\title{
Molecular analysis of late onset Huntington's disease
}

\author{
B Kremer, F Squitieri, H Telenius, S E Andrew, J Theilmann, N Spence, \\ Y P Goldberg, M R Hayden
}

\begin{abstract}
Late onset Huntington's disease is characterised by onset of symptoms after the age of 50 and is usually associated with a milder course. We have analysed the CAG trinucleotide repeat within the HD gene in 133 late onset patients from 107 extended families. The median upper allele size for the CAG repeat was 42 with a range of 38 to 48 repeats. A significant negative correlation $(r=-0.29, p=0.001)$ was found between the length of repeat and age of onset for the total cohort. However, for persons with age of onset greater than 60 , no significant correlation was found. In addition, no significant correlation was found between age of onset and size of the lower allele and the sex of the affected parent or grandparent. There was no preponderance of maternal descent for late onset cases in this series. This study shows that variation in repeat length only accounts for approximately $7 \%$ of the variation in age of onset for persons beyond the age of 50 and clearly shows how with increasing onset age the effect of the repeat length on this onset age seems to diminish.

( Med Genet 1993;30:991-5)
\end{abstract}

For most affected persons Huntington's disease (HD) usually begins around 40 years of age with personality change, clumsiness, and a movement disorder and progresses inexorably to death approximately 17 years from the time of onset. ${ }^{12}$ Many affected persons, however, do not follow this expected natural history. For a small proportion of patients (approximately $5 \%$ ), onset occurs before the age of 20 (juvenile HD) with a clinical presentation dominated by intellectual decline, rigidity, and a more rapidly progressive course. ${ }^{12}$ The proportion of patients with late onset HD (onset of symptoms after 50 years of age) varies between 10 and $25 \%$ in different studies..$^{3-9}$ In general, these patients with later onset $\mathrm{HD}$ have a less progressive course, often with a milder choreiform disorder and, initially, a less prominent intellectual decline. ${ }^{121011}$

While paternal transmission has been shown to be more frequent in persons with juvenile $\mathrm{HD},{ }^{12}$ it has also been proposed that there is a preponderance of female transmission in persons with late onset illness, ${ }^{1213}$ although others have failed to reproduce this finding. ${ }^{14} 15$

The vast majority of patients with HD have been found to have an expansion of a CAG trinucleotide repeat in a new gene located within 4 p16.3. ${ }^{16}$ Different investigations have shown a highly significant correlation between the CAG repeat length and age of onset of HD. ${ }^{17-19}$ In our own initial series the CAG repeat length accounted for approximately $50 \%$ of the variation in age of onset in that cohort $\left(r^{2}=0 \cdot 49\right) .{ }^{19}$ Further analysis of juvenile onset cases showed that the repeat length was the major factor associated with the age of onset of juvenile HD $\left(r^{2}=0 \cdot 73\right) .^{20}$ Thus it appears that in juvenile onset cases the association between the CAG repeat length and age of onset is stronger than in patients with adult onset.

In this study of 133 patients with late onset HD from 107 separate families, we have investigated the influence of repeat length on age of onset in persons with late onset HD. Based on previous studies, we hypothesised that in these patients CAG repeat length would show a weaker correlation with onset than seen in patients with earlier onset. We show that there is a significant association between CAG length and age of onset in late onset HD, but that this correlation is indeed weaker than for earlier onset patients. Furthermore, in patients with onset over 60 , no significant association between CAG length and age of onset was found. In this cohort of HD patients with late onset, there was no predominance of maternal transmission.

\section{Methods}

PATIENT SELECTION

We have collected DNA and clinical details where possible on approximately 1100 affected persons with HD. Those patients who clearly had onset at 50 years or more and for whom DNA was readily available were chosen for study $(n=137)$. A total of 95 of these patients was previously included as part of our analysis of a cohort of 360 patients. ${ }^{19}$

\section{DNA ANALYSIS}

Genomic DNA was isolated from leucocytes by standard extraction procedures. ${ }^{21}$ PCR amplification of the HD CAG repeat was performed with primers HD344 (5' CCTTCGAGTCCCTCAAGTCCTTC $3^{\prime}$ ) and HD482 (5' GGCTGAGGAAGCTGAGGAG $\left.3^{\prime}\right)$. PCR conditions were $2 \mathrm{mmol} / 1 \mathrm{MgCl}_{2}$, $50 \mathrm{mmol} / 1 \mathrm{KCl}, 20 \mathrm{mmol} / 1 \mathrm{Tris}, \mathrm{pH} 8 \cdot 4,3.5 \%$ formamide, $15 \%$ glycerol, $200 \mu \mathrm{mol} / 1$ of each dNTP, $0.5 \mu \mathrm{mol} / \mathrm{l}$ of each primer, $15 \mathrm{nmol} / 1$ end labelled primer, and 1.25 U of Taq DNA polymerase per $25 \mu \mathrm{l}$ reaction. Thermal cycling conditions were $95^{\circ} \mathrm{C}$ for three minutes, followed by 30 cycles of $94^{\circ} \mathrm{C}$ for one
Received 12 August 1993 Revised version accepted 27 September 1993. 
Table 1 Clinical and genetic data of late onset cohort.

\begin{tabular}{llccc}
\hline & & $\begin{array}{c}\text { Total group } \\
(\mathbf{n}=133)\end{array}$ & $\begin{array}{c}\text { One proband } \\
\text { per nuclear } \\
\text { family } \\
(\mathbf{n}=112)\end{array}$ & $\begin{array}{c}\text { One proband } \\
\text { per extended } \\
\text { family } \\
(\mathbf{n}=107)\end{array}$ \\
\hline Sex & Female & 69 & 54 & 54 \\
Sex of affected parent & Male & 64 & 58 & 53 \\
Sex of affected & Female & 49 & 43 & 39 \\
grandparent & Female & 62 & 50 & 13 \\
Age of onset & Male & 20 & 16 & 19 \\
Upper allele size & Mean & 20 & 20 & \\
(n=133) & Range & $57 \cdot 1$ & & \\
Lower allele size & Range & $50-85$ & & \\
(n=133) & Median & $38-48$ & & \\
\hline
\end{tabular}

minute, $62^{\circ} \mathrm{C}$ for one minute, $72^{\circ} \mathrm{C}$ for one minute, with a final extension at $72^{\circ} \mathrm{C}$ for seven minutes using a PE 9600 thermal cycler. PCR products were resolved as previously described ${ }^{1922}$ on $6 \%$ polyacrylamide gels.

\section{STATISTICAL ANALYSIS}

The association between age of onset and CAG size was investigated as described in a large cohort with predominantly adult onset disease. ${ }^{19}$ Age of onset was log transformed allowing the treatment of an exponential function as an intrinsically linear model. For correlation analysis, initially all affected patients were analysed as one group. As observations of multiple family members cannot be considered truly independent observations, the statistical analysis was repeated with one affected member per nuclear family and with one affected member per extended family.

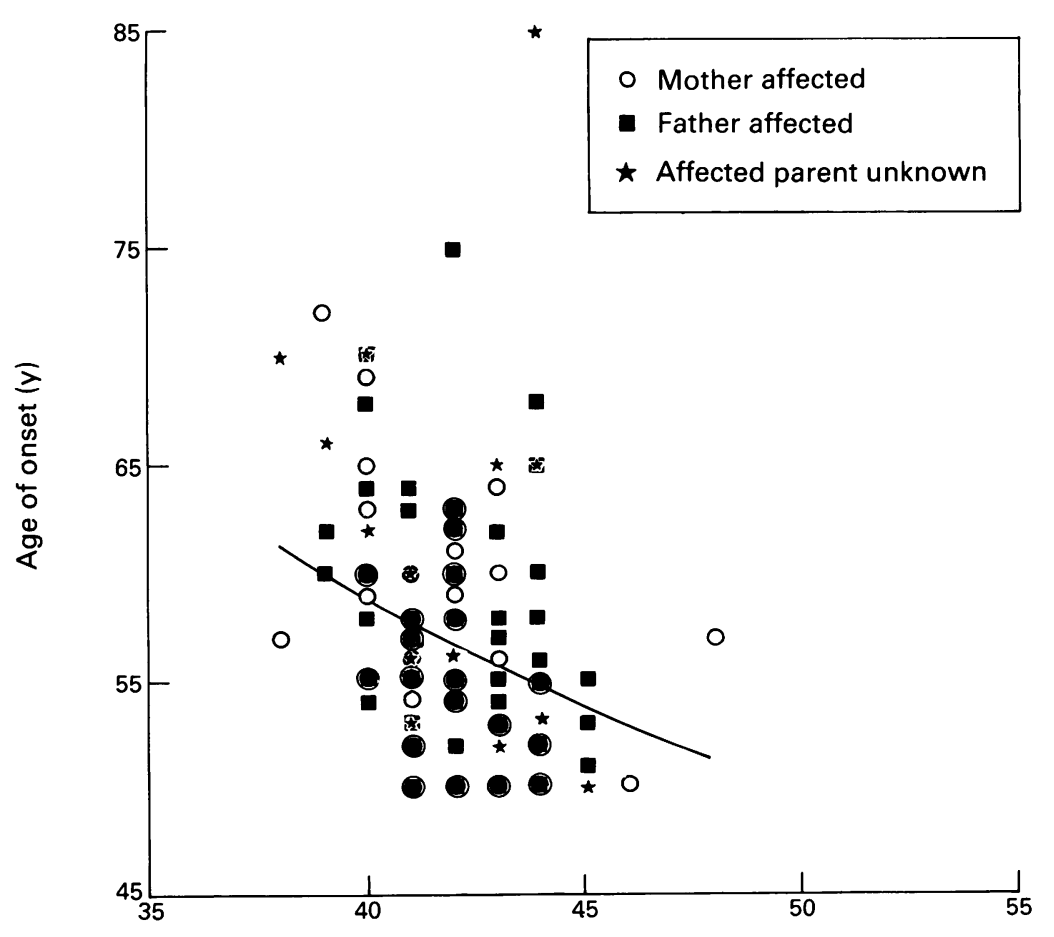

CAG repeat length

Figure 1 Age of onset $v$ CAG repeat length in 133 late onset patients ( $r=-0 \cdot 29$, $p=0.001$, for log transformed age of onset $)$. Note that overlapping values are represented as a single dot.

\section{Results}

SEX OF AFFECTED PARENTS OF LATE ONSET PATIENTS

A total of 137 patients from 111 separate families was included in this cohort (table 1). The sex of the affected parent was confirmed in 111 affected persons. No significant alteration in the expected sex distribution of the probands was determined. Furthermore, there was no preponderance of maternal descent for late onset patients in this cohort (table 1).

REPEAT LENGTH IN LATE ONSET PATIENTS

An allele in the range previously determined ${ }^{19}$ for affected patients with HD was seen in 133 of 137 persons. The median repeat length was 42 with a range of 38 to 48 (table 1 , fig 1). There were four patients who did not have any detectable expansion, as all four had two alleles in the normal range but had clinical features compatible with the diagnosis of HD. Two late onset patients without repeat expansion are from families in whom at least two other affected persons have repeat expansion in the range seen in patients with $\mathrm{HD}(18 / 43,17 / 47$ and $25 /$ $50,17 / 43$ for lower and upper alleles in affected persons in each of these families, respectively). The presence of two normal sized alleles in these two affected subjects from two families where expansion has been shown in other affected persons is unexplained at present. These subjects may represent phenocopies and were excluded from subsequent analysis.

\section{ASSOCIATION BETWEEN REPEAT LENGTH AND} AGE AT ONSET

A significant negative correlation $(n=133$, $r=-0.29, p=0.001$ ) was found between the length of the repeat and the age of onset for the total cohort of late onset patients (fig 1, table 2). However, for the subgroup of patients with age of onset above $60(n=42)$, no significant correlation was found between the size of the upper allele and the age of onset $(n=42$, $r=-0.05$, NS). Similar findings were obtained when the analysis was restricted to one member per nuclear family or one member of the extended family respectively (table 2). This analysis was also conducted on affected HD members in our whole cohort with onset between 20 and 50 years and those with onset below 20. A higher correlation coefficient between CAG repeat and age at onset is evident as the age of onset decreases (table 2).

No significant correlation could be found between age at onset and the size of the lower allele, nor between the age of onset and sex of affected parent or grandparent. Furthermore, neither the sex of the affected parent or grand-

Table 2 Correlation between age at onset and $C A G$ length.

\begin{tabular}{lrcr}
\hline & No & $\mathrm{r}$ & $\mathrm{p}$ value \\
\hline Onset $>60 \mathrm{y}$ & 42 & -0.05 & 0.759 \\
Onset $>50 \mathrm{y}$ & 133 & -0.29 & $<0.001$ \\
Onset $20-50$ & 308 & -0.60 & $<105$ \\
Onset $<20$ & 42 & -0.86 & $<10^{7}$ \\
\hline
\end{tabular}




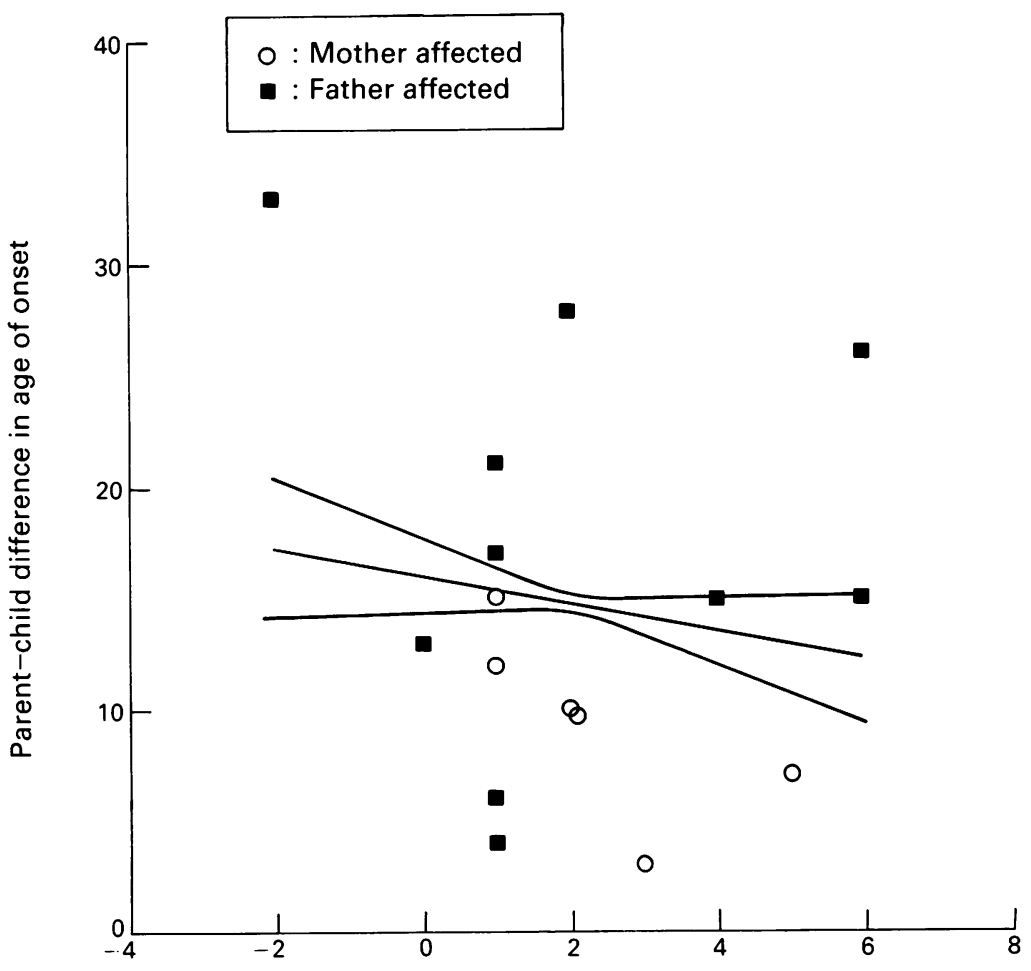

Parent-child difference in CAG length

Figure 2 Parent-child difference in age of onset $v$ parent-child difference in $C A G$ repeat length for 15 late onset patients and their children. The regression line and its $95 \%$ confidence interval are indicated, showing a non-significant association.

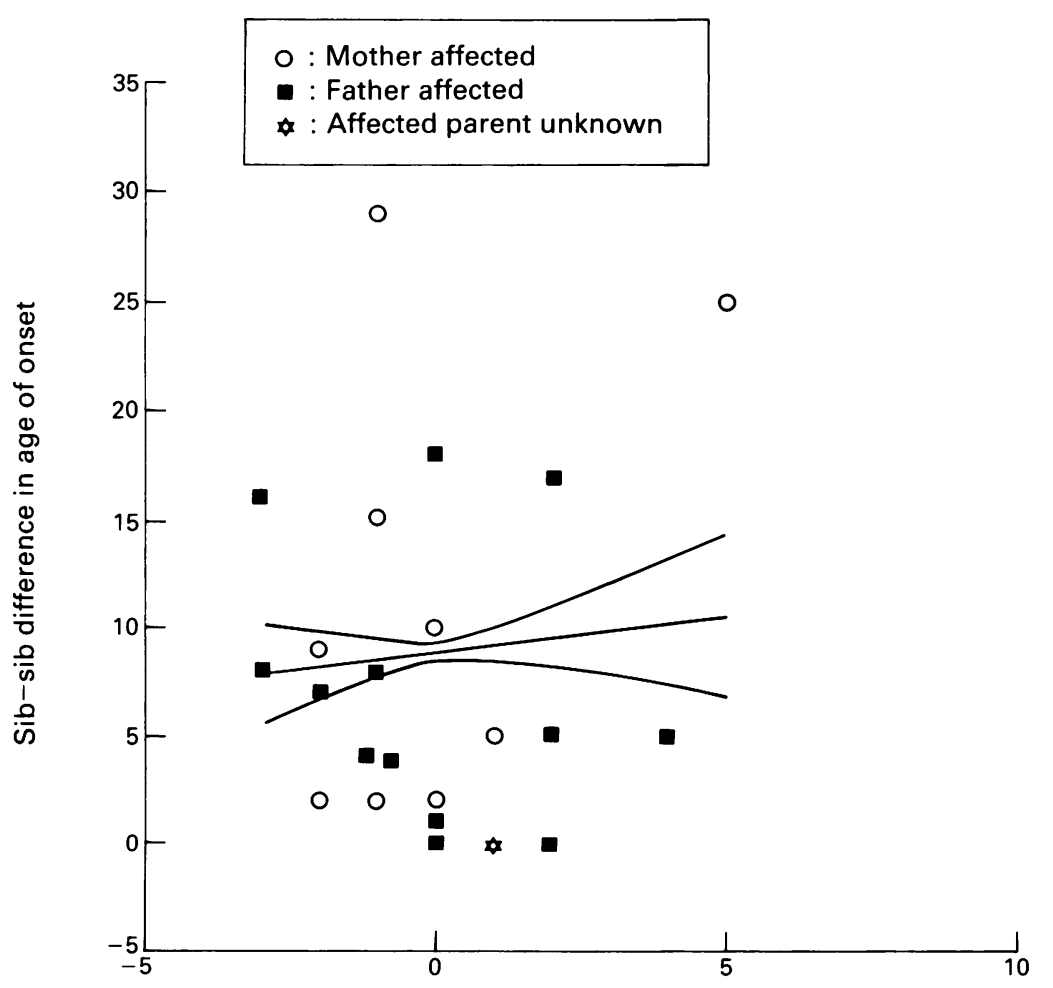

Sib-sib difference in CAG length

Figure 3 Sib-sib difference in age of onset $v$ sib-sib difference in $C A G$ repeat length for 23 late onset patients and their sibs. Data of the younger sib are subtracted from data of the older sib. The regression line and its $95 \%$ confidence interval are indicated, showing a non-significant association. parent showed any significant association with the CAG repeat size.

RELATIONSHIP BETWEEN REPEAT LENGTH AND AGE OF ONSET IN FIRST DEGREE AFFECTED RELATIVES

There were 16 parent/child pairs for study from 15 nuclear families in whom the parent had late onset and from whom clinical data and DNA were available. In all instances, there was no difference in allele size of greater than six repeats. However, there were marked variations in age of onset (up to 33 years) in these offspring compared to their parents (fig 2). In eight parent-child pairs in whom the difference in age of onset was 15 years or greater, the transmitting parent was the father in seven instances (fig 2). No significant correlation between repeat length differences and variation in age at onset were seen in these parentchild pairs ( $n=16, r=-0.16, N S$ ) (fig 2). Therefore, while there are obvious differences in age of onset between the parent and the child, the repeat length difference did not appear to account for these variations in the age of onset.

There were 20 families available for sib-sib analysis. These included 17 with data available on two sibs and three with data on three affected sibs. Therefore 23 affected sib pairs were analysed. CAG repeat length varied by five repeats or fewer in the sibs (fig 3). In $17 \mathrm{sib}$ pairs, differences in age at onset were less than 10 years with a familial tendency to late age of onset in these sibs (fig 4). However, in six sibships, differences in age of onset were between 10 and 29 years with CAG repeat length differences of less than five.

\section{Discussion}

In this study we have shown that there is a significant association between repeat length and age of onset of patients who first manifest signs and symptoms of HD after the age of 50 . However, for the 42 patients with age of onset after the age of 60 in the study, no significant association could be found between age at onset and size of the upper allele.

We have previously reported on the correlations between repeat length and age of onset in a predominantly adult cohort of patients with HD and in a separate cohort of persons with juvenile onset. ${ }^{1920}$ In that cohort of adult patients with HD (mean age of onset 41.5 years) the repeat length accounted for approximately $50 \%$ of the variation in age of onset. ${ }^{19}$ However, for the cohort with juvenile onset $\mathrm{HD}$, the length of the repeat accounted for approximately $73 \%$ of the variation in age of onset. ${ }^{20}$ This trend is borne out in this study, with the variation in repeat length accounting for only approximately $7 \%$ of the variation in age of onset in the whole cohort beyond the age of 50, without any significant influence on the age of onset in persons beyond the age of 60 . Thus, it is clear from these three studies that the contribution of repeat length to age of onset is greatest for those with earliest onset 
A

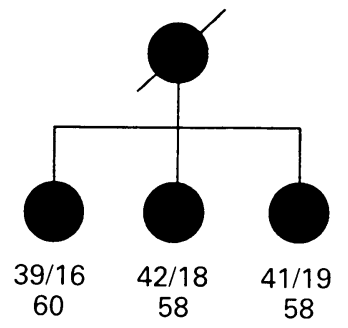

C

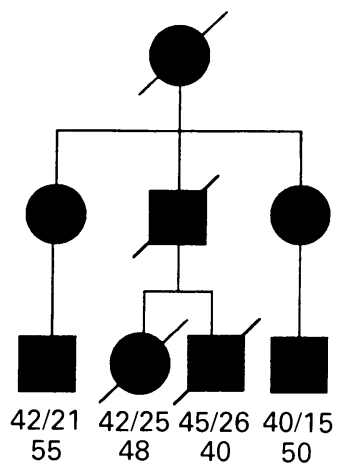

58

58
Allele size Onset age 55
B

Allele size Onset age

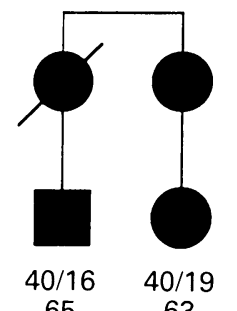

Figure 4 Family studies show familial aggregation of late onset in some family members within nuclear and extended families.

and appears to have less significance for persons with onset after 60 years.

There are several possible explanations to account for this variable contribution of CAG repeat length to age of onset. One potential bias which would have greater impact for persons with late onset $\mathrm{HD}$ who have repeat lengths in the low affected range for HD (median 42 repeats in this study) would be created by the recent recognition that the CCG repeat adjacent to the CAG repeat in the $5^{\prime}$ area of the $\mathrm{HD}$ gene may also display some polymorphism (Andrew et al, submitted). PCR primers used to assess the CAG repeat length flank both the CAG and CCG repeats. ${ }^{1922}$ Since this polymorphism may vary by only a few triplets, this would have most influence on the estimated repeat length in persons with repeat lengths in the lower range. If this occurred randomly on HD chromosomes, this might result in an underestimate of the association between CAG length and the clinical manifestations of HD. This would only be true, however, if there were no linkage disequilibrium between the CCG repeat length and the CAG repeat length mutations with HD.

Another explanation for these data might be related to the impact of CAG repeat expansion on the pathogenesis of HD. It is clear that the vast majority of patients with $\mathrm{HD}$ have expanded CAG repeats. ${ }^{17-19}$ However, what is not known is how CAG repeat length relates to the biology of the disease. If the effect of CAG repeats on the protein product in the pathogenesis of HD were related to the size of the repeat length, it might be expected that the most expanded repeats would have the greatest effect on the production of the mutant gene product and might be expected to result in more severe manifestations of the illness. As

repeat length has already been associated with age of onset, ${ }^{17-19}$ it is most likely that the size of the repeat in some way directly affects the gene product resulting in a more severe phenotype. Earlier onset could reflect a more pronounced effect of the abnormal gene product on neuronal cells. The relative contribution of other factors which might contribute to the pathogenesis of HD would decrease with increased CAG size.

In this study, we show no preponderance of maternal transmission in persons with late onset HD. There have been at least three previous studies which adopted a similar methodology in terms of ascertainment of patients and have resulted in conflicting findings (table 3). Interestingly, if one combines the data from all studies, while there is a small preponderance of affected mothers for late onset cases, this does not reach any statistical significance. There are numerous potential pitfalls in such studies which in part may account for the conflicting findings. A bias may be introduced by the omission of many cases where the sex of the affected parent was not known. The sex of the affected parent of the late onset cases was not known in approximately one third of the cases in the study by Myers et al, ${ }^{12}$ but only in $14 \%$ in the report of Farrer and Conneally ${ }^{13}$ and in $17 \%$ in this study. This bias would, however, only be in effect if the unknown sex of the transmitting parent was not random. Therefore, this is unlikely to be a major factor accounting for these different findings. Another potential bias might be counting a parent more than once if there are multiple affected sibs. However, when this particular issue was looked at specifically in the data set of this study, this did not appear to influence the results. A third factor that should be considered is whether these different study groups were strictly compatible in terms of age of onset. If preponderance of maternal descent for HD was mainly seen in those with latest age at onset, inclusion of a large number of very late onset cases would bias these results. To explore this, we examined our cohort of 42 with onset after 60 years. In this group, the number of affected mothers was 15 and affected fathers 17 , while the sex of the transmitting parent was unknown in 10 persons. Even in this selected group there is no evidence for preponderance of maternal descent.

Our study included 16 parent/child pairs for whom DNA analysis and also age of onset data were available. Similarly, data were available for 23 pairs of affected sibs. In some families there is familial aggregation of late onset cases both within nuclear families and in extended pedigrees (fig 4). This supports findings from

Table 3 Sex of affected parents of late onset cases.

\begin{tabular}{|c|c|c|}
\hline & Father & Mother \\
\hline $\begin{array}{l}\text { Myers et al }{ }^{12} \\
\text { Farrer and Conneally } \\
\text { Adams } \\
\text { This study }\end{array}$ & $\begin{array}{l}13 \\
35 \\
63 \\
62\end{array}$ & $\begin{array}{l}32 \\
56 \\
53 \\
49\end{array}$ \\
\hline Total & 173 & 190 \\
\hline
\end{tabular}


this and previous studies, ${ }^{202324}$ that have suggested there is familial aggregation of age of onset both within nuclear and extended families for both early and late onset illness. These factors can be taken into account as broad principles when counselling at risk family members of affected persons. These data strongly indicate that there are familial factors present in these families, besides CAG repeat size, influencing the onset and severity of the illness.

Previous studies have appropriately commented on the difficulties in the designation of age of onset in affected persons (summarised in reference $1, \mathrm{pp}$ 45-9). This may be particularly apparent for late onset cases where the disease may be milder in presentation. This factor may play some role in reducing the strength of the association between CAG repeat length and age at onset in late onset cases. Recent studies $^{17-19}$ determining the relationship between trinucleotide repeat length and age of onset clearly may underestimate the relationship between such variables in view of the fact that the estimates of age of onset may show considerable variation around the true age of onset. Nevertheless, it is evident from this study that as the age of onset increases, the impact of repeat length on this clinical onset appears to show less influence. Factors other than the CAG repeat length are likely to play a more important role in determination of age of onset for persons with onset beyond the age of 50 . These might include other genetic factors as well as possible environmental factors that are influencing the expression of the abnormal gene. Understanding these factors will no doubt provide valuable insights into the pathogenesis of this devastating disorder.

We thank members of our research group for their helpful comments. This work was supported by the MRC (Canada), the Canadian Genetic Disease Network, and the Huntington Society of Canada. YPG is a Sandoz postdoctoral fellow. BK and $\mathrm{HT}$ are MRC postdoctoral fellows. FS is supported by the Instituto
Scientific Sanatrix. MRH is an established investigator for the BC Children's Hospital.

1 Hayden MR. Huntington's chorea. New York: SpringerVerlag, 1981.

2 Harper PS. Huntington's disease. London: Saunders, 1991.

3 Davenport CB, Muncey EB. Huntington's chorea in relation to heredity and eugenics. $A m \quad \mathcal{f}$ Insanity 1916;73:195-222.

4 Bell J. Huntington's chorea. In: Treasury of human inheritance. Vol 4, part 1. London: Cambridge University Press, ance.

5 Reed TE, Chandler JH, Hughes EM, Davidson RT. Huntington's chorea in Michigan: demography and genetics. Am f Hum Genet 1958;10:201-25.

6 Cameron D, Venters GA. Some problems in Huntington's chorea. Scot Med f 1967;12:152-6.

7 Oliver JE. Huntington's chorea in Northamptonshire. $\mathrm{Br} \mathcal{f}$ Psychiatry 1970;116:241-53.

8 Walker DA, Harper PS, Wells CEC, et al. Huntington's chorea in south Wales: a genetic and epidemiological study. Clin Genet 1981;19:213-21.

9 Hayden MR. Huntington's chorea in South Africa. PhD thesis, University of Cape Town, 1979.

10 Myers RH, Sax DS, Schoenfeld M, et al. Late onset of Huntington's disease. $\mathcal{F}$ Neurol Neurosurg Psychiatry 1985;48:530-4.

11 Faught E, Falgout MS, Leli DA. Late-onset variant of Huntington's chorea. Southern Med f 1983;76:1266-70.

12 Myers RH, Goldman D, Bird ED, et al. Maternal transmission in Huntington's disease. Lancet 1983;i:208-10.

13 Farrer LA, Conneally MP. A genetic model for age at onse in Huntington disease. Am $\mathcal{F}$ Hum Genet 1985;37:350-7.

14 Went LN, Vegter-van der Vlis M, Bruyn GW. Paternal transmission in Huntington's disease. Lancet 1984;i:1100-2.

15 Adams P. Statistical analysis of age of onset in Huntington's disease. $\mathrm{PhD}$ thesis, Georgia State University, 1986.

16 Huntington Disease Collaborative Research Group. A Huntington Disease Collaborative Research Group. A
novel gene containing a trinucleotide repeat that is novel gene containing a trinucleotide repeat that is
expanded and unstable on $\mathrm{HD}$ chromosomes. Cell expanded and

17 Duyao MP, Ambrose C, Myers R, et al. Trinucleotide repeat length instability and age of onset in Huntington's disease. Nature Genet 1993;4:387-92.

18 Snell RG, MacMillan JC, Cheadle JP, et al. Relationship between trinucleotide repeat expansion and phenotypic variation in Huntington's disease. Nature Genet 1993;4:393-7.

19 Andrew SE, Goldberg YP, Kremer B, et al. The relationship between trinucleotide (CAG) repeat length and clinical features of Huntington's disease. Nature Genet cal features of

20 Telenius H, Kremer HPH, Theilmann J, et al. Molecular analysis of juvenile Huntington disease: the major influence of paternal descent on (CAG) repeat length influence of paternal descent on
Hum Mol Genet 1993;2:1535-40.

21 Kunkel LM, Smith KD, Boyer SH, et al. Analysis of human $\mathrm{Y}$ chromosome specific reiterated DNA in chromosome variants. Proc Natl Acad Sci USA 1977;74:1245-9.

22 Goldberg YP, Andrew SE, Clarke LA, Hayden MR. A PCR method for accurate assessment of trinucleotide repeat expansion in Huntington disease. Hum Mol Genet 1993;2:635-6.

23 Brackenridge CJ. Familial correlations for age at onset and age at death in Huntington's disease. $\mathcal{F}$ Med Genet 1972;9:23-32.

24 Pericak-Vance MA, Elston RC, Conneally PM, Dawson DV. Age-of-onset heterogeneity in Huntington disease families. Am f Med Genet 1983;14:49-59. 